\title{
THE COLICINE TYPING OF COLIFORM BACILLI IN THE STUDY OF CROSS-INFECTION IN UROLOGY
}

\author{
BY \\ K. B. LINTON \\ From the Department of Pathology, United Bristol Hospitals
}

(RECEIVED FOR PUBLICATION SEPTEMBER 17, 1959)

\begin{abstract}
Three hundred and fourteen strains of coliform bacilli (79 Klebsiella and 235 E. coli), mostly isolated from urine, were investigated for their production of, or sensitivity to, certain colicines.

Only one Klebsiella strain showed colicine activity, but in 204 of the E. coli strains colicine production or sensitivity was demonstrated.

Colicine typing was of considerable value in epidemiological investigations of post-operative urinary infections due to $E$. coli.
\end{abstract}

Antibiotic-like substances which are produced by many Gram-negative bacilli and are able to inhibit the growth of related strains were first described by Gratia (1925), and were later called "colicines" (Gratia and Frédéricq, 1946).

Recently, tests based on the ability of bacteria to produce colicines, or on their sensitivity to colicines produced by other strains, have been employed in epidemiological studies of infection by intestinal pathogens, e.g., Shigella sonnei, and strains of Escherichia coli isolated during outbreaks of gastro-enteritis (Frédéricq, Betz-Bareau, and Nicolle, 1956; Shannon, 1957; Abbott and Shannon, 1958).

This paper describes the use of colicine tests in a study of cross-infection of the urinary tract, mainly among urological patients in this hospital (Miller, Gillespie, Linton, Slade, and Mitchell, 1958).

\section{Methods}

The basic techniques were those described by Frédéricq, Thibault, and Gratia (1946) and employed, with some modifications, by Shannon (1957) for colicine-typing strains of $E$. coli which caused gastroenteritis, and by Abbott and Shannon (1958) for typing strains of Shigella sonnei.

From a survey of the properties of colicine production and colicine sensitivity of 200 coliform strains isolated during our studies of urinary infections and of 13 strains obtained by $\mathrm{Dr}$. A. Mayr-Harting (University of Bristol) from Professor P. Frédéricq (University of Liége), standard sets of strains were selected for use in the typing methods described below. Ten colicine-sensitive strains of $E$. coli isolated in this department (nine from urine and one from faeces) were chosen for the colicine production tests and were designated "strain 1, $2 \ldots$ 10." Of the 10 colicine producers used for the sensi- $\vec{\bullet}$ tivity tests, seven were Professor Frédéricq's strains 8 (Ca 7, Ca 18, Ca 31, Ca 42, Ca 53, Ca 62, $\mathrm{K} \mathrm{235);}$ the other three were isolated in this department. The 10 producer strains were designated by the letters "A, B . . . J."

Medium.-Ten per cent. blood agar plates, contain-

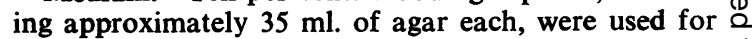
all tests. (The agar was deeper than in normal plates $\overrightarrow{\overrightarrow{0}}$ to avoid exhaustion of the nutrient ; $10 \%$ blood was 3 incorporated in the medium as a source of catalase $\vec{\partial}$ to eliminate inhibition due to any hydrogen peroxide which might be formed during the test (Gardner, 1950).)

(1) Screening Tests for Detection of Colicine Production-Early in the work, it was found that test strains which produced any demonstrable colicines invariably inhibited one particular standard sensitive $₹$ strain (strain 4). Accordingly, strain 4 was used to $ᄋ$ provide a screening test for colicine production by unknown test strains. If the screening test was negative, no further production test was done; if positive, the test strain was put up against the full $N^{\circ}$ set of standard sensitive strains (see method 2).

The screening test was made by inoculating test 0 strains (six per plate), radiating like the spokes of a $\mathrm{\omega}$ wheel, on blood agar plates. The plates were then incubated for 48 hours at $37^{\circ} \mathrm{C}$. A disc of filter 0 paper was then placed in the lid of the Petri dish and saturated with chloroform; the dish containing the $\stackrel{\mathbb{D}}{+}$ medium was then replaced and the plate left inverted on the bench for two hours. By this means, the surface layers of the streaks of bacterial growth were $\frac{\vec{D}}{\mathbb{D}}$ killed by the chloroform vapour. Next, the streaks $\stackrel{?}{\oplus}$ of growth were scraped to the centre of the plate with $\stackrel{D}{\Omega}$ a clean glass slide and this small area of agar removed. More chloroform was then added to keep the filter paper moist, and the plate left closed for a 
further hour. The filter paper was then removed and the plate exposed to the air for two hours, with the lid open and the surface of the medium downwards.

A 1-in-4 dilution of an overnight broth culture of $E$. coli strain 4 (indicator strain) was then prepared and inoculated in a circular streak, crossing the centre of the line of growth of each of the test strains. After incubation overnight, positive results were shown by inhibition of the indicator strain (Fig. 1).
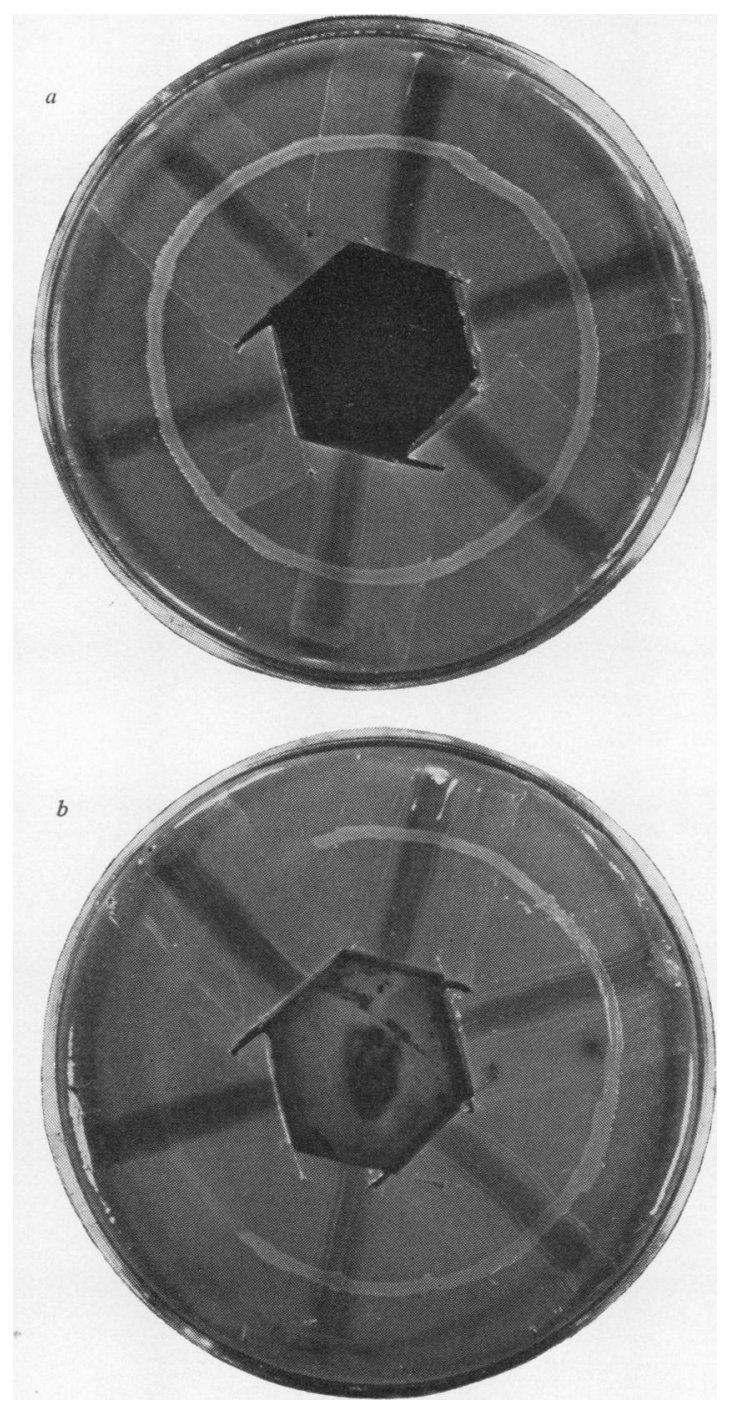

Fig. 1.-Six test strains were grown on each plate in the positions shown by the darkened agar. After treatment with chloroform, the lines of growth were scraped into the centre of the plate and this small area of agar removed. A ring of inoculum (sensitive strain 4) was then added. (a) No colicine activity. The smooth and constant growth of sensitive strain produced by the special inoculation wire can be seen. (b) Two of the strains produced colicines.

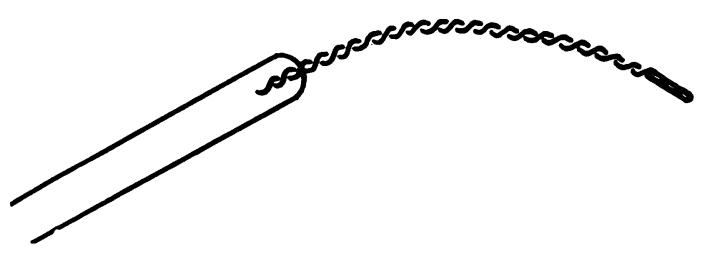

FIG. 2.-Inoculating wire.

In the methods described in the literature, a 3-4 $\mathrm{mm}$. wire loop is used for the inoculation of indicator strains on test plates, but, in our experience, such loops frequently gave irregular and tapering streaks of growth. However, a twisted double platinum wire with the tip bent to serve as a nib, when dipped in the dilution of indicator culture, was found to hold sufficient fluid around the two twisted wires to supply a constant inoculum from the tip over six or seven inches of agar surface (see Figs. 1 and 2). When inoculating a large number of plates, it was unnecessary to flame the wire between each plate, but it was flamed before being introduced into a broth culture of a different organism (Abbott and Shannon, 1958).

(2) Typing by Colicine Production.-The coliform strain to be tested (test strain) was inoculated heavily across a plate to give a confluent streak of growth (primary streak). It has been found quite satisfactory to inoculate two test strains per plate. After incubation at $37^{\circ} \mathrm{C}$. for 48 hours, the plates were treated with chloroform as previously described for the screening tests, the lines of growth in this case being scraped to one end and removed together with a small piece of agar. After final aeration, the plates were then inoculated with the standard set of colicinesensitive bacilli (strains 1-10). One-in-four dilutions of overnight broth cultures of these organisms were used, and they were inoculated across the plate at right angles to the line of the primary streak. The plates were incubated at $37^{\circ} \mathrm{C}$. overnight, and examined next day.

If no colicine was formed, active against the indicator strains, all streaks grew across the plate as parallel confluent lines of growth. Where detectable colicines were produced, areas of inhibition of one or more of the standard sensitive strains could be seen, distributed symmetrically around the original position of the primary streak (Fig. 3). The inhibition zone sizes were recorded, using the notation described by Abbott and Shannon (1958) as follows:

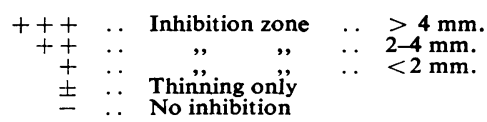

Subscript R.-Presence of numerous resistant colonies in zone of inhibition

Subscript D.-A form of inhibition consisting of a central area of growth with a zone of inhibition on either side. 


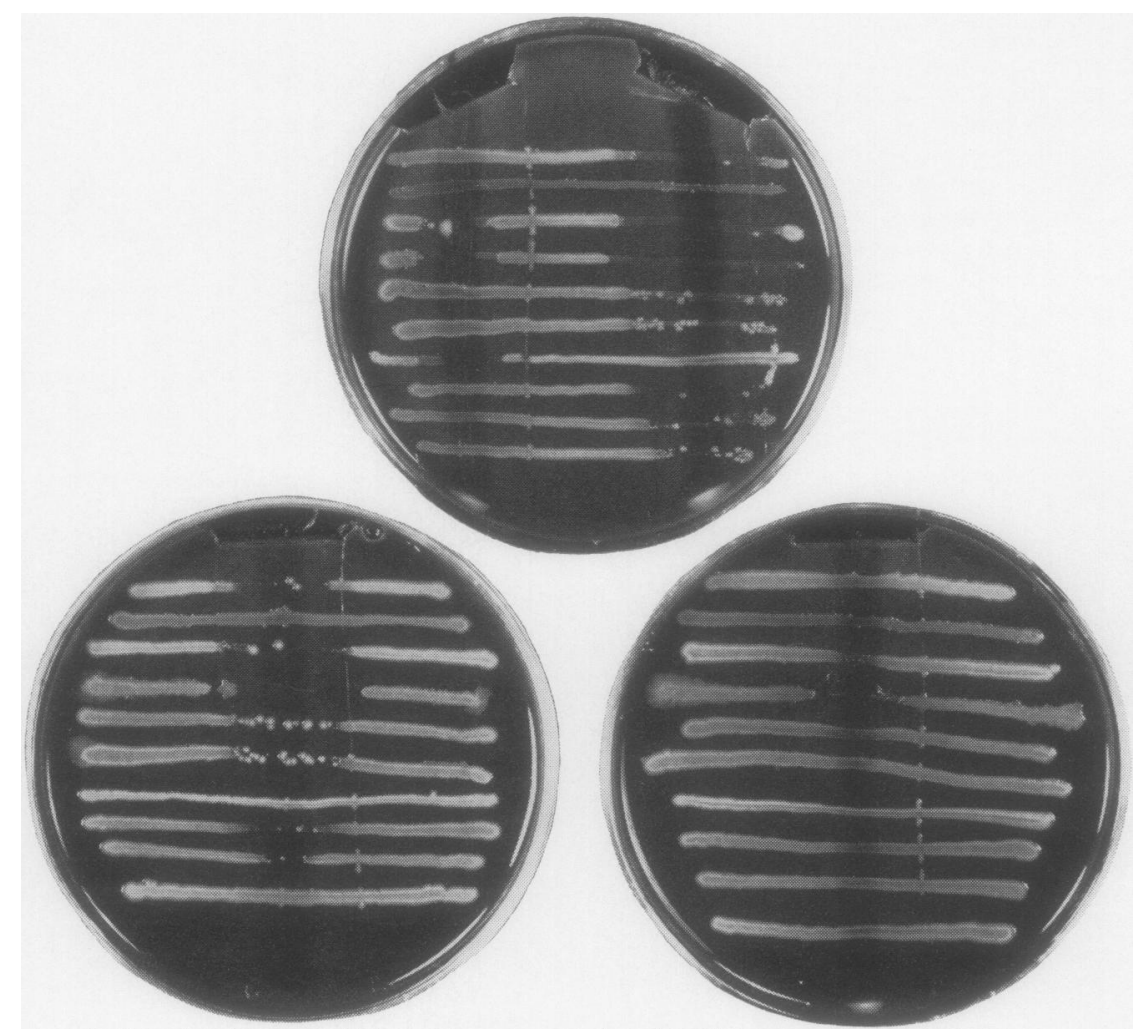

FIG. 3.-Three test plates for typing by colicine production.

(3) Typing by Sensitivity to Colicines.-The standard colicine-producing strains (A, B . . . J) were grown (two streaks per plate) and treated by the same techniques as the test strains in the production method (2). Overnight broth cultures of the organisms to be tested were then diluted 1 in 4 and inoculated, 10 per plate, on each of the five primarily inoculated plates. Results were normally recorded after 18 hours' incubation using the same notation as for colicine-production typing.

(4) Controls.-The standard strains were checked during each series of colicine tests by testing the colicine-producing set of strains against the standard colicine-sensitive set.

\section{Distribution of Colicine-producing and} Colicine-sensitive Strains.-Three hundred and fourteen coliform strains, isolated during investigations of urinary infection, have so far been examined by the typing methods described. The organisms were obtained from the following sources: 11 strains from swabs of urethra or perineum, 33 strains from faeces, 269 strains from urine, and one strain from blood.
Seventy-nine of the cultures were members of the genus Klebsiella, and only one showed any activity, being colicine sensitive. The remaining 235 organisms were strains of $E$. coli ; $97(41 \%)$ produced demonstrable colicines, 172 (73\%) showed sensitivity to one or more of the colicines produced by the standard strains, and $31(13 \%)$ showed neither kind of activity (Table I).

TABLE I

COLICINE ACTIVITY OF 235 STRAINS OF E. COLI

\begin{tabular}{|c|c|c|}
\hline \multirow{2}{*}{$E$. coli Cultures } & \multicolumn{2}{|c|}{ Activity Demonstrated } \\
\hline & Colicine Production & Colicine Sensitivity \\
\hline $\begin{array}{r}31 \\
32 \\
107 \\
65\end{array}$ & $\begin{array}{l}\bar{t} \\
\bar{t}\end{array}$ & $\begin{array}{l}\overline{-} \\
\overline{+} \\
+\end{array}$ \\
\hline Total 235 & 97 & 172 \\
\hline
\end{tabular}

Designation of "Types."-The strains displayed a large number of different colicine patterns, and, in order to record comparisons of strains from different sources, a simple method of designation was adopted for local use. For this, the readings 
from colicine test plates were grouped into the following three categories:

Positive + Complete inhibition, no account being taken of the size of the inhibition zone or of small numbers of resistant colonies

Doubtful \pm Thinning of growth above the line of the primary streak or a small inhibition zone containing many resistant colonies

Negative - Normal growth

The pattern of activity of each test strain was recorded as a series of figures or letters indicating the standard strains against which positive reactions were obtained. Brackets were used when reactions were doubtful.

Examples:

(1) Production typing, E. coli (Lab. No. 2004).

\begin{tabular}{|c|c|c|c|c|c|c|c|c|c|c|}
\hline & \multicolumn{10}{|c|}{ Indicator Strains } \\
\hline $\begin{array}{l}\text { Activity . } \\
\text { Simplified } \\
\text { pattern }\end{array}$ & $\begin{array}{c}1 \\
++t_{R} \\
+\end{array}$ & $\begin{array}{l}2 \\
-\end{array}$ & $\mid \begin{array}{c}3 \\
++ \\
+\end{array}$ & $\begin{array}{c}4 \\
++ \\
+\end{array}$ & $\begin{array}{c}5 \\
++_{k} \\
+\end{array}$ & $\begin{array}{c}6 \\
+{ }_{+R} \\
+\end{array}$ & $\begin{array}{l}7 \\
- \\
-\end{array}$ & $\begin{array}{l}8 \\
-\end{array}$ & $\begin{array}{l}9 \\
- \\
-\end{array}$ & $\begin{array}{l}10 \\
- \\
-\end{array}$ \\
\hline
\end{tabular}

This pattern was recorded as $1 / 3,4,5,6$.

(2) Sensitivity typing, E. coli (Lab. No. 2706).

\begin{tabular}{l|c|c|c|c|c|c|c|c|c|c}
\hline & \multicolumn{8}{|c}{ Colicine-producing Strains } \\
\cline { 1 - 6 } & A & B & C & D & E & F & G & H & I & J \\
$\begin{array}{c}\text { Activity. } \\
\text { pattern }\end{array}$ & +++ & - & - & - & $+\mathbf{R}$ & ++ & - & - & - & - \\
\hline
\end{tabular}

This pattern was recorded as $A^{\prime}(E) F$.

So far, 30 different patterns of colicine production and 55 different patterns of colicine sensitivity have emerged from these systems of typing.

Most of the patterns were exhibited by only a few strains, but one of the production patterns and one of the sensitivity patterns were frequently encountered; 29 strains gave the production pattern $1 / 3 / 4 / 5 / 6 / 8 / 9 / 10$ and 52 strains had the sensitivity pattern A. The widespread occurrence of these patterns was not thought to be the result of frequent cross-infection by a few established "hospital" organisms, since strains with these patterns were isolated between 1955 and 1959 from both urine and faeces and, during some investigations not reported in this paper, from the faeces of normal persons outside hospital. Moreover, by a variety of methods, comparisons of these strains with those of Professor Frédéricq indicated that organisms of production pattern $1 / 3 / 4 / 5 / 6 / 8 / 9 / 10$ produced colicine $\mathrm{V}$, the most active and common of the colicines, and that organisms of sensitivity pattern A were inhibited only by that strain of the standard set which produced colicine $\mathrm{V}$.

Use of Colicine Typing in Tracing Routes and Sources of Cross-infection.-Colicine typing, by the methods described, has a serious limitation in that it is largely inapplicable to the Klebsiella group of organisms which are responsible for much of the cross-infection among urological patients in this as in other hospitals (Ørskov, 1952 and 1954). However, with coliform bacilli other than strains of Klebsiella, the methods, by virtue of the high specificity of colicine activity, are very useful in tracing routes and sources of infection.

The property of colicine sensitivity has been found by Abbott and Shannon (1958) to be unreliable as an epidemiological marker in studies of Shigella sonnei, but Shannon (1957) has suggested that the unreliability may be due to the production of resistant variants in vivo by the action of the colicine-producing organisms which Gratia and Weerts (1946) have shown to be present in almost all specimens of faeces. On the other hand, we have found colicine sensitivity to be a stable and reproducible property of a strain isolated repeatedly from the same patient; but this may be because many of our coliform strains were isolated in pure culture from urine and not from faeces.

Some examples of the use of colicine typing are given below.

(1) On February 26, 1957, Mr. A. L. was admitted from another hospital with an indwelling catheter. The urine was heavily infected with $E$. coli (Lab. No. 3587) and Streptococcus faecalis. After operation on March 1, 1957, the patient remained on open drainage (urine draining into an open non-sterile vessel at the bedside) for at least a fortnight. Mr. G. H. was admitted to the next bed on March 4, 1957, and his urine remained sterile until prostatectomy on March 6, 1957, after which an apparatus for closed aseptic drainage and irrigation was fitted (Miller et al., 1958). His urine was sterile next day, but on the same evening the apparatus became disconnected in the ward and was reassembled by the nursing staff, a complication which, experience has shown, usually leads to infection. On March 8, 1957, his urine was found to contain $E$. coli (Lab. No. 3626), and the infection which developed was sufficiently severe to require antibiotic treatment. Unlike any other strains of $E$. coli isolated during the three years of our investigations, both cultures 3587 and 3626 were of the same colicine-sensitivity pattern, $A / B / C /(F) / J$, and were also identical in biochemical characteristics and antibiotic sensitivity. It is clear, therefore, that Mr. G. H. became infected by the coliform strain which had been introduced into the ward by Mr. A. L. 
and had been allowed to proliferate in the urine receptacle near Mr. G. H.'s bed. The infection may have occurred when the closed drainage apparatus was disconnected, probably from the contaminated hands of a nurse.

(2) On January 7, 1958, Mr. P. was cystoscoped in another hospital. His urine contained large numbers of coliform bacilli (Lab. No. 4900). After use, the cystoscope was immersed horizontally in $1 / 2,000$ mercuric oxycyanide solution for 11 minutes before rinsing in sterile water, a procedure which, we have found, cannot be relied upon to disinfect cystoscopes (Miller et al., 1958). The same instrument was then used for the examination of another patient, Mr. C. Coliform bacilli (Lab. No. 4903) were also grown from this patient's urine and from a rectal swab taken at operation (Lab. No. 4907). Specimens of urine were obtained from both patients the following day. Cultures from urine taken during and after operation from Mr. P. showed no change in bacterial flora throughout. Culture of the first urine passed after operation by $\mathrm{Mr}$. C., however, revealed two types of colony; one resembled that grown from his urine before operation, but the other appeared to be different. On the following day, Mr. C.'s urine, on culture, yielded large numbers of the new type of colony, and this organism had biochemical properties identical with those of strain 4900 which had been isolated from the urine of Mr. P. Neither showed any colicine activity, in contrast to the pre-operative urinary and faecal strains of $\mathrm{Mr}$. C. which were clearly different. The results of the colicine tests are given below, and supported the belief that the infection was transferred on an inadequately disinfected cystoscope.

EXAMINATION FOR COLICINE ACTIVITY

\begin{tabular}{|c|c|c|c|}
\hline & & Production & Sensitivity \\
\hline $\begin{array}{l}\text { Mr. P. } \\
\text { Mr. C. }\end{array}$ & $\begin{array}{l}\text { Urine } 4900 \\
\begin{cases}\text { Pre-operative urine } 4903 & \ldots \\
\text { Faeces 4907 (10 different } \\
\text { colonies tested) } \\
\text { Post-operative urine } 4937 \quad \ldots\end{cases} \end{array}$ & $\begin{array}{l}- \\
\pm \\
+\end{array}$ & $\begin{array}{l}\overline{+} \\
\overline{-}\end{array}$ \\
\hline
\end{tabular}

(3) A urethral swab, taken from Mr. R. C. immediately before undergoing transurethral prostatectomy (November 13, 1957), yielded $E$. coli (Lab. No. 4343). The urine specimens taken before, during, and immediately after operation were sterile, but an infection started on the second post-operative day, caused by $E$. coli (Lab. No. 4351). The infecting strain and the strain isolated from the patient's urethra before operation proved to have identical patterns of colicine production and sensitivity (production pattern $2 / 3 / 4 / 7$, sensitivity pattern $A$ ). It would appear that small numbers of coliform bacilli, present in the urethra before operation, were introduced into the bladder during instrumentation and subsequently caused urinary infection.

(4) Mrs. S. O. underwent a gynaecological operation on February 5, 1959. Within 18 hours of operation, her urine had become infected by a strain of $E$. coli (Lab. No. 6735), production pattern $1 / 3 / 4 / 5 / 6 / 8 /(9)$ and sensitivity pattern $B / G / J$. A strain with identical patterns was isolated from a rectal swab taken at operation. The infection was probably caused by an organism originating in the patient's own intestine, possibly as a result of contamination during catheterization.

(5) Mrs. R. D. developed a urinary infection after several days of catheter drainage following a gynaecological operation on March 2, 1959. Culture of the urine yielded two different coliform strains. Neither produced colicines, and only one showed sensitivity, giving pattern $\mathrm{C} / \mathrm{I}$. Coliform bacilli, clearly different from both of the urinary strains, were isolated from the rectal swab (Lab. No. 6878) taken at operation. These also did not produce colicines but were of sensitivity pattern $A$. In this case, therefore, the infecting organisms probably came from the environment.

It is a pleasure to express my gratitude to Dr. W. A. Gillespie for his help and encouragement, and to the Board of Governors of the United Bristol Hospitals for a grant towards the cost of this research.

I would also like to thank Dr. A. Mayr-Harting and Professor P. Frédéricq for some cultures, and Dr. M. T. Parker, Dr. J. D. Abbott, and Mr. R. Shannon for teaching me their techniques of colicine typing.

\section{REFERENCES}

Abbott, J. D., and Shannon, R. (1958). J. clin. Path., 11, 71. Frédéricq.,P., Betz-Bareau, M., and Nicolle, P.(1956). C. R. Soc. Biol. (Paris), 150, 2039

- Thibault, J., and Gratia, A. (1946). Ibid., 140, 1035.

Gardner, J. F. (1950). Brit. J. exp. Path., 31, 102.

Gratia, A. (1925). C. R. Soc. Biol. (Paris), 93, 1040.

Gratia, A. (1925). C. R. Soc. Biol. (Paris), 93, 104 and Frédéricq, P. (1946). Ibid., 140,1032.

Miller, A., Gillespie, W. A., Linton, K. B., Slade, N., and MitcheK J. P. (1958). Lancet, 2, 608.

Ørskov, I. (1952). Acta. path. microbiol. scand., Suppl. 93, 259.

- (1954). Ibid., 35, 194.

Shannon, R. (1957). J.med. lab. Technol., 14, 199. 